Werner, Klaus Ulrich (Hrsg.): Bibliotheken als Orte kuratorischer Praxis. De Gruyter Saur, 2021.

ISBN 978-3-11-067372-2. € 69,95

Besprochen von Prof. Dr. Ursula Georgy: Technische Hochschule Köln, Institut für Informationswissenschaft, Gustav-Heinemann-Ufer 54, D-50968 Köln, E-Mail: ursula.georgy@th-koeln.de

https://doi.org/10.1515/bfp-2021-0004

Das Buch „Bibliotheken als Orte kuratorischer Praxis“ ist ein weiteres aus der Serie der Bibliotheks- und Informationspraxis des De Gruyter Verlages, und - um es vorweg zu nehmen - es wird dem Anspruch der Praxis auch voll gerecht.

\section{Relevanz des Themas}

Den Begriff Kuratieren assoziieren die meisten sicher (noch) mit Kunst und Kultur, wobei damit meist die Fähigkeit gemeint ist, Kunst- und Kulturgegenstände auszuwählen, um sie in einem Raum, z. B. Museen, Kunsthallen und Kunstvereinen so zu präsentieren, dass sie in ihrer Konstellation zusammentreffen und zusammenpassen oder dass sie in Narrationen eingebunden werden. Der Begriff hat aber in den letzten Jahren eine starke Wandlung erfahren, teilweise wird er inzwischen fast inflationär verwendet. Kuratorisches Handeln hat heute in starkem Maße eine gesellschaftliche Funktion mit hohen kollaborativen und partizipatorischen Anteilen erfahren, wobei alle Beteiligte unterschiedliche Rollen einnehmen können und sich gegenseitig ergänzen. Und damit hat Kuratieren selbstverständlich auch für Bibliotheken im Rahmen von „Open“ eine wichtige Funktion erlangt und kann noch deutlich ausgebaut werden. Daher kommt ein Buch zu dem Thema zur rechten Zeit.

\section{Beiträge}

Der Herausgeber Klaus Ulrich Werner hat die insgesamt 14 Beiträge seines Handbuches fünf Themenbereichen zugeordnet:

I. Theorie und Praxis des Kuratierens,

II. Fallstudien,

III. Data Curating/Content Curating,

IV. Bürgerwissenschaften in Bibliotheken sowie

V. Räume kuratieren.

Das Buch beginnt mit einer Einführung und der Frage „Heute schon kuratiert?“, in der der Herausgeber einstimmt auf die neue Rolle und Funktion des Kuratierens, wobei insbesondere das Einbeziehen von Kund ${ }^{\star}$ innen und Bürger*innen und der Gesellschaft betont werden. Dabei sind nach Meinung von Klaus Ulrich Werner vor allem die Fähigkeiten und Kompetenzen jeder/jedes Einzelnen im Rahmen des Kuratierens gefragt, um im Sinne der Bibliothek Fachdiskurse zu führen, Projekte zu realisieren, die ohne Kollaboration und Partizipation nicht möglich gewesen wären. In der Einleitung gibt Klaus Ulrich Werner zudem einen kurzen Einblick in die verschiedenen Beiträge des Buches. Bereits hier fällt auf, dass zahlreiche Autor^innen eine hohe Affinität zu Kunst und Kultur haben, aber gleichzeitig Bibliotheken eng verbunden sind bzw. über einen bibliothekarischen Hintergrund verfügen. Genau dadurch gelingt auch der Transfer des Kuratierens im Sinne der bibliothekarischen Praxis.

Im Folgenden werden bewusst nur wenige Beiträge konkret besprochen, vielmehr geht es darum, wie das Thema in dem Buch erfasst und dargestellt wird und was das Buch vermittelt.

Das Buch beginnt mit einem sehr umfangreichen Beitrag von Jan-Tillmann Rierl zum erweiterten Kuratierungsbegriff und der bibliothekarischen Praxis. Der Beitrag hat es sicher verdient, an dieser prominenten Stelle im Buch zu stehen, denn in ihm wird der erweiterte Begriff des Kuratierens unter sehr unterschiedlichen Aspekten ausführlich und tiefgehend behandelt. Aber braucht man diese Tiefe und Ausführlichkeit für das Buch? Ja und Nein. Ja, 
weil der Beitrag sehr fundiert erläutert, dass es nicht um die inflationäre Verwendung eines Begriffs geht, sondern darum, wie sich Kuratieren gewandelt hat und völlig neue Perspektiven für Bibliotheken und ihre Kunden eröffnet. Nein, weil man eigentlich darauf gespannt ist, wie Kuratieren in Bibliotheken in seiner modernen Form konkret erfolgt und gelebt wird, und da möchte man als Leser*in gern schnell zu den nächsten Beiträgen übergehen. Daher würde man sich für diesen Beitrag eine Art „Blinkist ${ }^{\text {* “ von }}$ wenigen Seiten wünschen. Aber für das tiefere Verständnis des Buches sollte man diesen Beitrag keinesfalls auslassen oder nur flüchtig lesen. Es folgt ein Interview des Herausgebers mit Friederike Hauffe, selbst Kuratorin. Es darf als mutig bezeichnet werden, in ein solches Buch auch Interviews zu integrieren, sind sie doch manchmal wenig fundiert. Aber die Fragen hier sind knapp und präzise gestellt, so dass die Antworten eine gute Ergänzung zum Einführungsbeitrag darstellen, wobei der persönliche Charakter im Sinne eines Fragegesprächs nicht zu kurz kommt. Vor allem aber schlägt Klaus Ulrich Werner in dem Beitrag einen Bogen zum Trendmanagement, indem er den Megatrend „Partizipation“ besonders hervorhebt - letztendlich der zentrale Aspekt des Buches.

Es folgen in Teil II insgesamt sechs Fallstudien. Einige der Projekte wurden bereits in anderen Kontexten publiziert und sind somit in der bibliothekarischen Community in weiten Teilen bekannt, z. B. Kunstbibliothek Sitterwerk, Münchner Stadtbibliothek, New York Public Library. Aber es sei vorweggenommen: Alle Beiträge überzeugen durch ihre klare Fokussierung auf das Kuratieren. Eine Zweitbzw. Mehrfachverwertung von Inhalten ist immer legitim, sofern neue Akzente und Schwerpunkte gesetzt werden. Und das ist bei allen Beiträgen der Fall.

In den Teilen III, IV und V werden Data Curating/ Content Curating, Bürgerwissenschaften und Räume mit Beiträgen u. a. zu Crowdsourcing, Citizen Science und Coworking in den Fokus gestellt. Die Teile III und V könnte man im Sinne des Managements von Bibliotheken bzw. des Innovationsmanagements auch dem Thema Open Innovation zuordnen. Und damit ist man auch wieder bei dem zentralen Aspekt des Buches angelangt. Kuratieren im weiteren Sinne bedeutet auch und vor allem die strategische Öffnung von Bibliotheken, um einen gesellschaftlichen Mehrwert durch das Leben einer Kultur der Partizipation und des Zusammenarbeitens zu schaffen. Für Bibliotheken erfordert dies in vielen Fällen eine Änderung des Mindset im Sinne eines strategischen Offenheitsverständnisses. Kuratieren im Sinne der Partizipation ist ein Paradigmenwechsel weg vom „abhängigen“ Kunden hin zum handlungsfähigen und souveränen Akteur. Alle Beiträge greifen diese Aspekte gut auf, auch wenn vielleicht in dem ein oder anderen Beitrag diese Besonderheit noch deutlicher und konkreter hätte hervorgehoben werden können. Aber wenn das Buch dabei hilft, auf eine Änderung des Mindsets Richtung Offenheit hinzuwirken, dann hat es seinen Sinn mehr als erfüllt.

\section{Fazit}

Es bleibt die Hoffnung, dass der Begriff des Kuratierens, der bei vielen sicher noch mit einem „klassischen“ Verständnis assoziiert wird, nicht vom Kauf/Lesen abhält. Aber das ist auch eine Frage des Marketings, wobei der Verlag gefordert ist, diese mögliche Zurückhaltung gegenüber diesem Thema bei potenziellen Leser`innen zu überwinden. Vielleicht wäre ein Untertitel wie z. B. „Partizipation in Bibliotheken" hierfür ein erster Ansatz gewesen.

Über die Reihenfolge der Beiträge in dem Buch kann man sicher streiten. Warum wurden z.B. die Fallstudien nicht den Themenbereichen zugeordnet? Aus Sicht der Rezensentin wäre dies gut möglich gewesen, und der Bogen zwischen Theorie und Praxis würde noch deutlicher. So kommt es insbesondere in den drei thematischen Teilen immer wieder zu Redundanzen zu den Fallbeispielen. Diese wären durch eine andere Ordnung vermeidbar gewesen. Mindestens aber hätte man sich Verweise auf die jeweiligen Beiträge - vice versa - gewünscht. Dies hätte die Überlegungen hinter der Auswahl der Autor`innen und der Themen seitens des Herausgebers noch einmal gestützt.

Das Buch entspricht dem hohen Standard des De Gruyter Verlags und ist sehr gut lesbar. Wurde vonseiten der Rezensentin immer wieder kritisiert, dass Fotos und Grafiken bei De Gruyter nicht durchgängig farbig gedruckt werden, so ist dieses „must have“ in diesem Buch erfreulicherweise umgesetzt. Fast will man sagen: endlich.

\section{Zielpublikum}

In dem Buch werden ausgewählte Beispiele kuratorischen Handelns in verschiedensten Bibliotheken präsentiert: Öffentlichen und wissenschaftlichen, aber auch Spezialbibliotheken, womit das Buch für viele unterschiedliche Zielgruppen von Interesse ist: Bibliotheken oder/und Bibliotheksabteilungen mit ihren Mitarbeiterinnen, aber auch Studierende, die sich mit dem Thema des Kuratierens intensiv(er) auseinandersetzen möchten bzw. müssen.

Dieses Buch ist ein Muss für alle Bibliotheken und es lohnt sich. 